\title{
A method for establishing a patient-derived xenograft model to explore new therapeutic strategies for esophageal squamous cell carcinoma
}

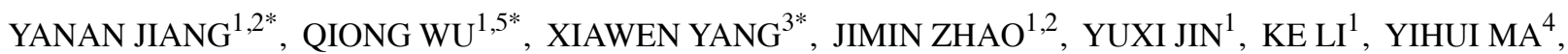 \\ XINHUAN CHEN $^{1,2}$, FANG TIAN ${ }^{1,2}$, SONG ZHAO $^{4}$, JINGLONG XU ${ }^{1}$, JING LU $^{1,2}$, \\ XUESHAN YIN $^{1}$, KANGDONG LIU ${ }^{1,2,5}$ and ZIMING DONG ${ }^{1,2}$
}

\author{
${ }^{1}$ Department of Pathophysiology, School of Basic Medical Sciences, Zhengzhou University; ${ }^{2}$ Henan Provincial Cooperative \\ Innovation Center for Cancer Chemoprevention; ${ }^{3}$ School of Life Sciences, Zhengzhou University, Zhengzhou, Henan 450001; \\ ${ }^{4}$ The First Affiliated Hospital of Zhengzhou University, Zhengzhou University, Zhengzhou, Henan 450052; \\ ${ }^{5}$ China-US (Henan) Hormel Cancer Institute, Zhengzhou, Henan 450008, P.R. China
}

Received August 30, 2015; Accepted October 10, 2015

DOI: $10.3892 /$ or.2015.4459

\begin{abstract}
Esophageal squamous cell carcinoma (ESCC) is the predominant histological type of esophageal carcinoma in China. The overall 5-year survival rate of ESCC patients is in the low range of $15-25 \%$. One important reason for the poor prognosis is that the underlying molecular mechanisms are unclear. Furthermore, the development of effective therapeutic strategies to improve patient outcome is needed. Animal models can be beneficial to analyze the molecular mechanisms as well as specific clinical therapeutic strategies for esophageal cancer. In recent years, patient-derived xenografts (PDXs) have been widely used in numerous types of cancers to investigate the basic mechanisms and to conduct preclinical research. Accumulating evidence indicates that the PDX model is an important tool for basic and clinical research. Herein, we successfully established 14 ESCC PDXs. These PDX models preserved the patient pathological characteristics and effectively reflected the patient biological heterogeneity. Cancers exhibit diverse growth rates and tumor texture, even more, they have different signaling pathways. The PDX model is a superior strategy for understanding the underlying molecular mechanisms of ESCC and for screening new therapeutic strategies for ESCC patients.
\end{abstract}

Correspondence to: Dr Ziming Dong or Dr Kangdong Liu, Department of Pathophysiology, School of Basic Medical Sciences, Zhengzhou University, 100 Kexue Avenue, Zhengzhou, Henan 450001, P.R. China

E-mail: dongzm@zzu.edu.cn

E-mail:kdliu@zzu.edu.cn

*Contributed equally

Key words: esophageal squamous cell carcinoma, patient-derived xenograft, signaling pathway

\section{Introduction}

Esophageal cancer (EC) is one of the most common malignant diseases worldwide, particularly in China, where a high incidence of EC is noted. Esophageal squamous cell carcinoma (ESCC) is the predominant histological type of esophageal carcinoma in China (1). Several treatment approaches, including surgery, radiotherapy and chemotherapy are used in the clinic. However, the overall 5-year survival rate remains dismal (2). Therapeutic resistance at a late stage of ESCC is one of the significant reasons for the low survival rate (3).

Recent research indicates that tumorigenic signaling pathways are involved in the processes of ESCC, including tumor cell growth, cell cycling, apoptosis, angiogenesis and invasion $(2,4)$. Hence, the key factors of signaling pathways can be effective therapeutic targets for the treatment of ESCC. Unlike breast cancer and non-small cell lung cancer (5-8), there is no reported data concerning the targeted therapy of ESCC. Therefore, there is needed to develop an effective strategy to investigate the mechanisms of esophageal carcinogenesis and explore therapeutic strategies for specific individuals to improve the prognosis of esophageal patients.

Animal models are appropriate tools to resolve both basic and clinical EC research problems. In recent years, patientderived xenografts (PDXs) have been used to evaluate the effectiveness of targeted treatments for different types of tumors, such as breast and non-small cell lung cancer. The response of PDXs to chemotherapy resembles the patient response in different clinical treatment trials $(9,10)$. Hence, PDX models provide a platform with which to study genetic and biological alterations as well as specific large-scale anticancer therapies for cancer patients (11).

In the present study, we established and characterized ESCC PDXs by transplanting 26 ESCC patient tumor specimens into severe combined immunodeficiency (SCID) mice. The 'success-rate' of the PDX models was 53.8\%. Tumor growth in the first, second and third passage was observed. The pathology and the CK5/6, p63 and p40 expression levels 
Table I. Clinical characteristics of the studied patients.

\begin{tabular}{|c|c|c|c|c|c|c|}
\hline No. & $\begin{array}{c}\text { Age } \\
\text { (years) }\end{array}$ & Gender & $\begin{array}{c}\text { Tumor } \\
\text { differentiation }\end{array}$ & $\begin{array}{l}\mathrm{TNM} \\
\text { staging }\end{array}$ & $\begin{array}{l}\text { Lymph node } \\
\text { metastasis }\end{array}$ & $\begin{array}{c}\text { Engrafted } \\
\text { mode }\end{array}$ \\
\hline EG1 & 63 & Male & Poor & T4N0M0 III & No & No \\
\hline EG2 & 64 & Male & Well-Moderate & T2N0M0 IIa & No & Yes \\
\hline EG3 & 74 & Female & Well-Moderate & T2N0M0 IIa & No & Yes \\
\hline EG4 & 60 & Female & Moderate-poor & T3N0M0 IIa & No & No \\
\hline EG5 & 61 & Male & Moderate & T2N0M0 IIa & No & Yes \\
\hline EG6 & 65 & Female & Moderate-poor & TINOM0 I & No & No \\
\hline EG7 & 60 & Female & Moderate & T3N2M0 III & Yes & No \\
\hline EG8 & 63 & Male & Moderate & T2N0M0 IIa & Yes & Yes \\
\hline EG9 & 66 & Male & Well-Moderate & T2N1M0 IIb & Yes & Yes \\
\hline EG10 & 62 & Female & Well-Moderate & T2N0M0 IIa & No & No \\
\hline EG11 & 65 & Male & Poor & T3N0M0 IIa & No & Yes \\
\hline EG12 & 65 & Female & Well-Moderate & T2N0M0 IIa & No & No \\
\hline EG13 & 63 & Male & Moderate & T3N0M0 IIa & No & Yes \\
\hline EG14 & 59 & Male & Moderate & T2N0M0 IIa & No & Yes \\
\hline EG15 & 60 & Male & Moderate & T2N1M0 IIb & Yes & No \\
\hline EG16 & 58 & Female & Moderate-poor & T3N1M0 III & Yes & No \\
\hline EG17 & 82 & Male & Moderate & T3N0M0 IIa & No & No \\
\hline EG18 & 52 & Male & Well-Moderate & T3N1M0 I-II & Yes & Yes \\
\hline EG19 & 70 & Male & Moderate & T1N1M0 II & Yes & Yes \\
\hline EG20 & 46 & Female & Moderate & T2NOM0 II & No & Yes \\
\hline EG21 & 68 & Female & Moderate-poor & T3N1M0 II-III & Yes & Yes \\
\hline EG22 & 60 & Male & Well & T2N0M0 II & No & No \\
\hline EG23 & 61 & Male & Moderate-poor & T3N1M0 II-III & Yes & No \\
\hline EG24 & 47 & Male & Well & T3N1M0 I & Yes & Yes \\
\hline EG25 & 69 & Male & Well & T2N0M0 I & No & Yes \\
\hline EG26 & 64 & Male & Moderate & T3NOMO II & No & Yes \\
\hline
\end{tabular}

in the patient samples of the first and third passages were detected and compared with each other by hematoxylin and eosin (H\&E), and immunochemical staining. In addition, tumor genetic signaling pathway kinases, including mTOR and p-mTOR (Ser2481), Stat1 and p-Stat1 (Tyr701), Stat3 and p-Stat3 (Tyr705), Akt1 and p-Akt (Ser473), Erk and p-Erk (Thr202/Tyr204) were detected in five PDX models by western blotting. These PDX models provided a platform to further understand the molecular mechanisms of ESCC and screen new specialized therapeutic regimens for ESCC patients in preclinical research.

\section{Materials and methods}

Patient tissue procurement. All 26 ESCC patients underwent surgical operations at the First Affiliated Hospital of Zhengzhou University (Zhengzhou, China), and ESCC tissues were obtained intraoperatively from October 2013 to October 2014. All patients had not received chemotherapy and radiotherapy prior to surgery. Written informed consent was provided by all patents for using the tissue samples. All research protocols were approved by the Research Ethics Committee of Zhengzhou University. Tissue histology was confirmed by two pathologists. Fresh harvested ESCC speci- mens were obtained from the edge of whole tumor tissues to maintain high active and low necrotic part of the tissues. All tissues were transported to the laboratory in transport media [fetal bovine serum (FBS)-free, RPMI-1640 with penicillin and streptomycin].

Animals. Female CB17/SCID mice (6-8 weeks of age) with an average body weight of 18-20 g (Vital River, Beijing, China) were used in the study. Mice (4-5) were kept in a pathogen-free environment in light controlled rooms (12-h cycles) and were provided with food and water ad libitum.

Patient tumor xenografts. Tissue samples were placed in a Petri plate containing PBS with penicillin and streptomycin. The tissues were divided into three parts. One portion was implanted into SCID mice, the second portion was fixed in $10 \%$ formalin and the third portion was used for protein extraction. The mice were anesthetized using $0.3 \mathrm{ml}$ of $0.4 \%(\mathrm{w} / \mathrm{v})$ pentobarbital sodium for every $20 \mathrm{~g}$ of body weight. Then, they were subcutaneously implanted with tissues of different weight and size $(0.10-0.12 \mathrm{~g}$ and $\sim 3 \mathrm{~mm})$. Animals were monitored periodically for their weight and tumor growth. Second passage was performed at a tumor size of $\sim 1,500 \mathrm{~mm}^{3}$. The tumor specimen was cut into three parts and followed the same 

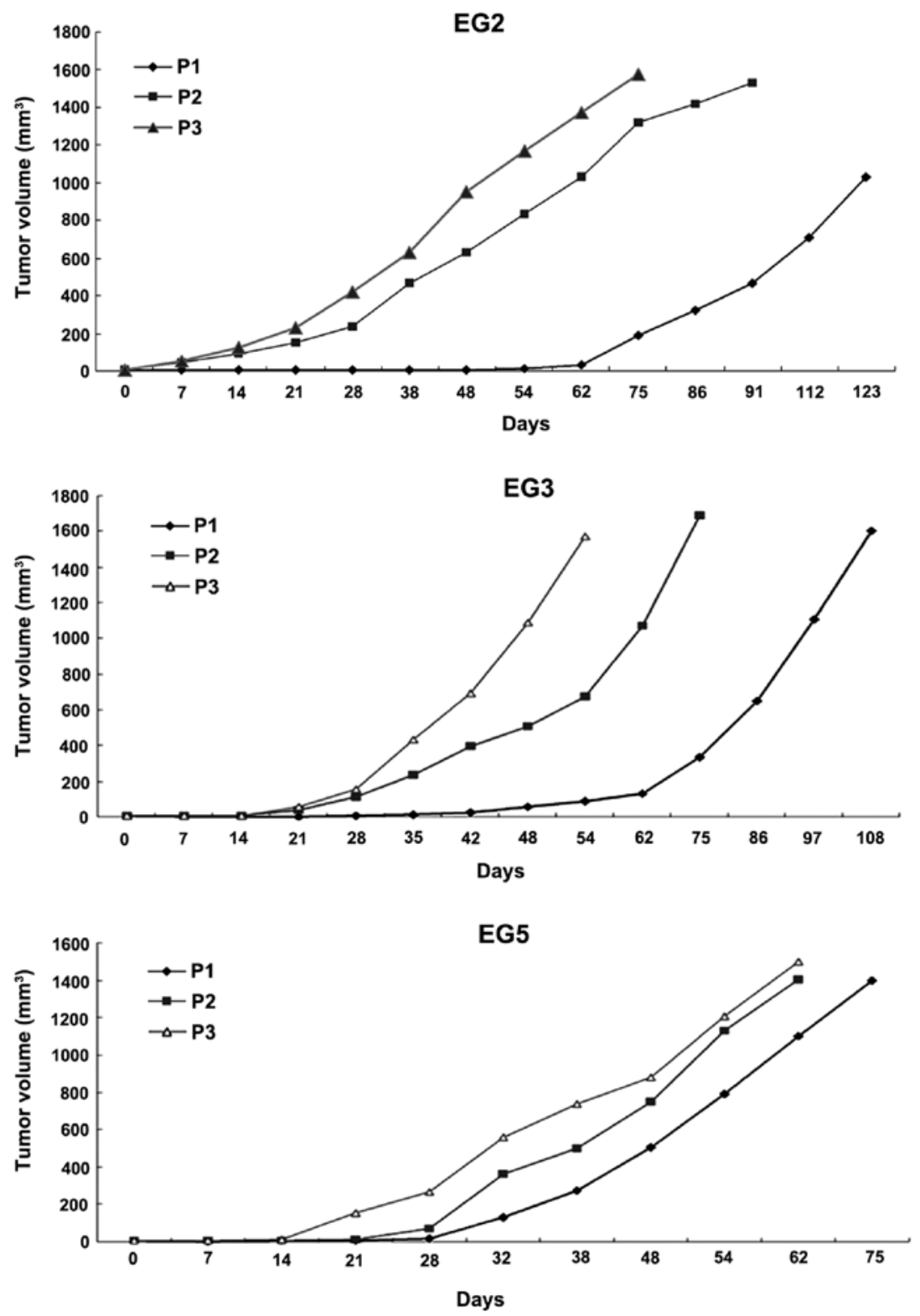

Figure 1. Growth curves of the ESCC xenografts in SCID mice. Growth curves of three passage xenografts (first, second and third passage) plotted as tumor volume over time. Xenografts were established from three ESCC patients (EG2, EG3 and EG5).

protocol as previously described. Successful tumor grafts were recognized as those with rapid tumor growth in vivo with more than three passages, and these were used for experimental studies.

Tumor growth measurements. The tumor xenograft size was measured with a Vernier caliper two times every week. The tumor volume was calculated using the formula: $\mathrm{V}=\mathrm{LD} \times(\mathrm{SD})^{2} / 2$, where $\mathrm{V}$ is the tumor volume, $\mathrm{LD}$ is the longest tumor diameter and SD is the shortest tumor diameter. Tumor growth curves were plotted as tumor volume.

Histological and immunohistochemical analysis. The second part of the tumor was embedded in paraffin for histopathologic examination and immunohistochemical analysis. All of the slides were stained with Harris hematoxylin after dewaxing the 5- $\mu \mathrm{m}$ sections with dimethylbenzene and were evaluated by two pathologists. Tissue sections were stained with antibodies against CK5/6, p63, p40 (Abcam, UK) and were incubated overnight at $4^{\circ} \mathrm{C}$. HRP-IgG secondary antibody was used at $37^{\circ} \mathrm{C}$ for $15 \mathrm{~min}$. Then diaminobenzidine (DAB) was used for detection. All slides were observed and measured by an Olympus microscope (Japan).

Western blotting. The PDX tissues were ground in liquid nitrogen and were lysed by tissue lysate. Next, tissue protein was extracted by centrifugation at $12,000 \mathrm{rpm}$ for $15 \mathrm{~min}$ at $4^{\circ} \mathrm{C}$. The protein $(50 \mu \mathrm{g})$ was separated on $10 \%$ SDS-polyacrylamide gel, and then transferred to a PVDF membrane at $90 \mathrm{~V}$ for $2 \mathrm{~h}$. The membrane was blocked with $5 \%$ non-fat milk for $60 \mathrm{~min}$. Then the membrane was incubated with mTOR, p-mTOR (Ser2481), Stat1, p-Stat1 (Tyr701), Stat3, p-Stat3 (Tyr705), Akt1, p-Akt (Ser473), Erk1/2 and p-Erk1/2 (Thr202/Tyr204) antibodies (Cell Signaling Technology, USA) overnight at $4^{\circ} \mathrm{C}$ 

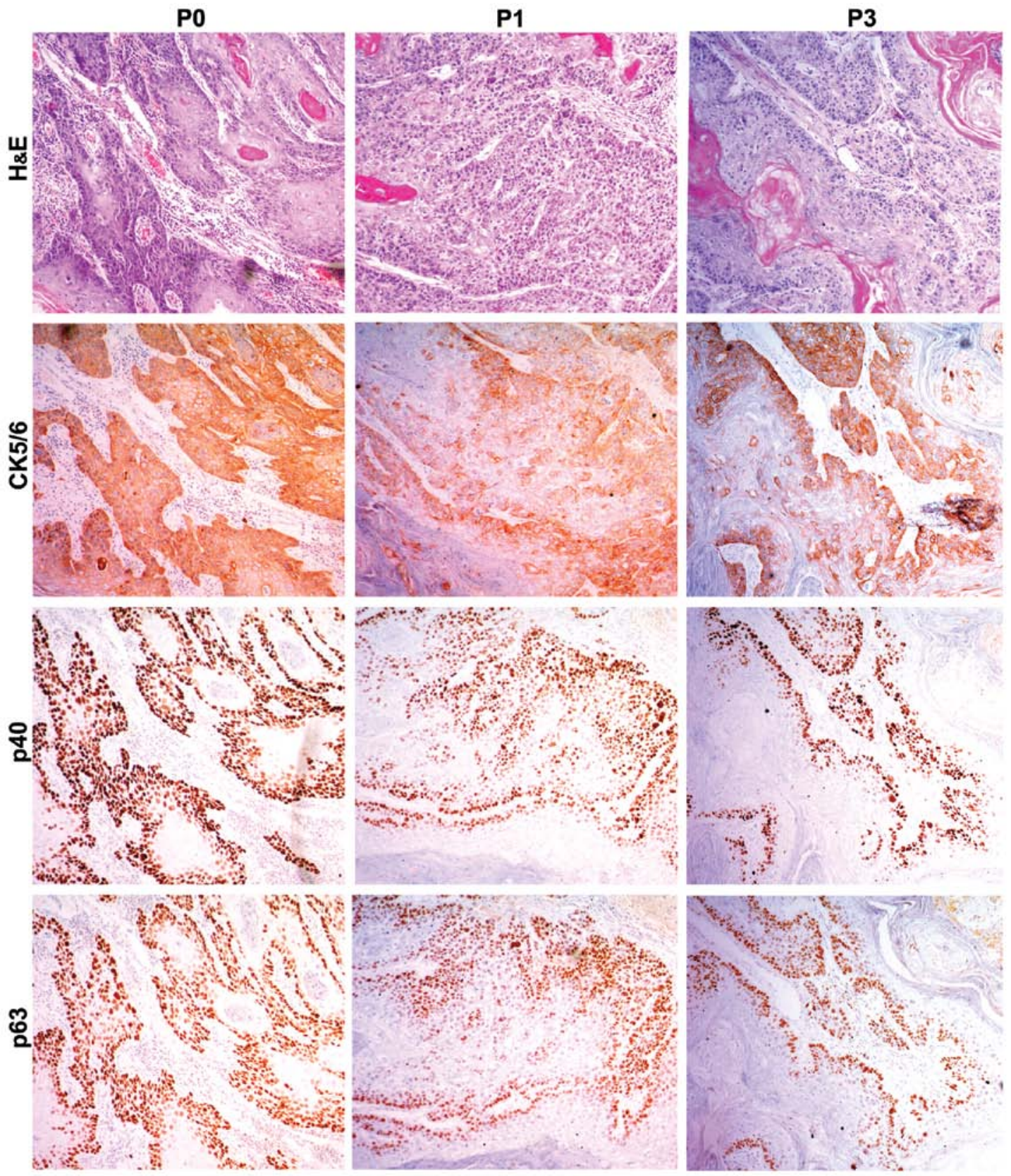

Figure 2. Comparative histology of the original patient tumors with first passage and third passage xenografts for sample EG2. The top row images show histological sections (H\&E) of the esophageal patient tissue (P0), the first (P1) and the third passage (P3) xenograft tissues in the SCID mice. The second, third and last row of images show immunohistochemistry for CK5/6, p40, p63 in the esophageal patient tissue (P0), and the first (P1) and third passage (P3) xenograft tissues, respectively (magnification, x100).

(all antibody were used at 1:1,000, Stat3 antibody was from mouse and other antibodies were all from rabbit). HRP-IgG secondary antibody was incubated at room temperature for $2 \mathrm{~h}$. Protein bands were visualized by an enhanced chemiluminescence detection kit (ECL; Pierce). The PVDF membranes were scanned by ImageQuant LAS 4000 and analyzed by ImageJ software v2.1.4.7.

Statistical analysis. All statistical analyses were performed using SPSS statistical software 17.0. Experimental values were reported as mean \pm standard error of the mean. One-way analysis of variance was used for statistical analysis. $\mathrm{P}<0.05$ was considered to indicate a statistically significant result.

\section{Results}

Clinical characteristics of the patients. In the present study, 26 ESCC subjects underwent surgical resection. The subjects consisted of 17 men and 9 women ranging from 46-82 years of age (62.6 \pm 7.4 years). The subjects did not have any apparent distant metastases, and none had been previously treated. In regards to differentiation, 2 samples were established from 3 well differentiated samples, 4 were from 6 wellmoderate differentiated samples, 7 were from 10 moderately differentiated samples, only 1 sample was established from 5 moderate-poorly differentiated samples while no sample was established from 2 poorly differentiated samples. Among these 

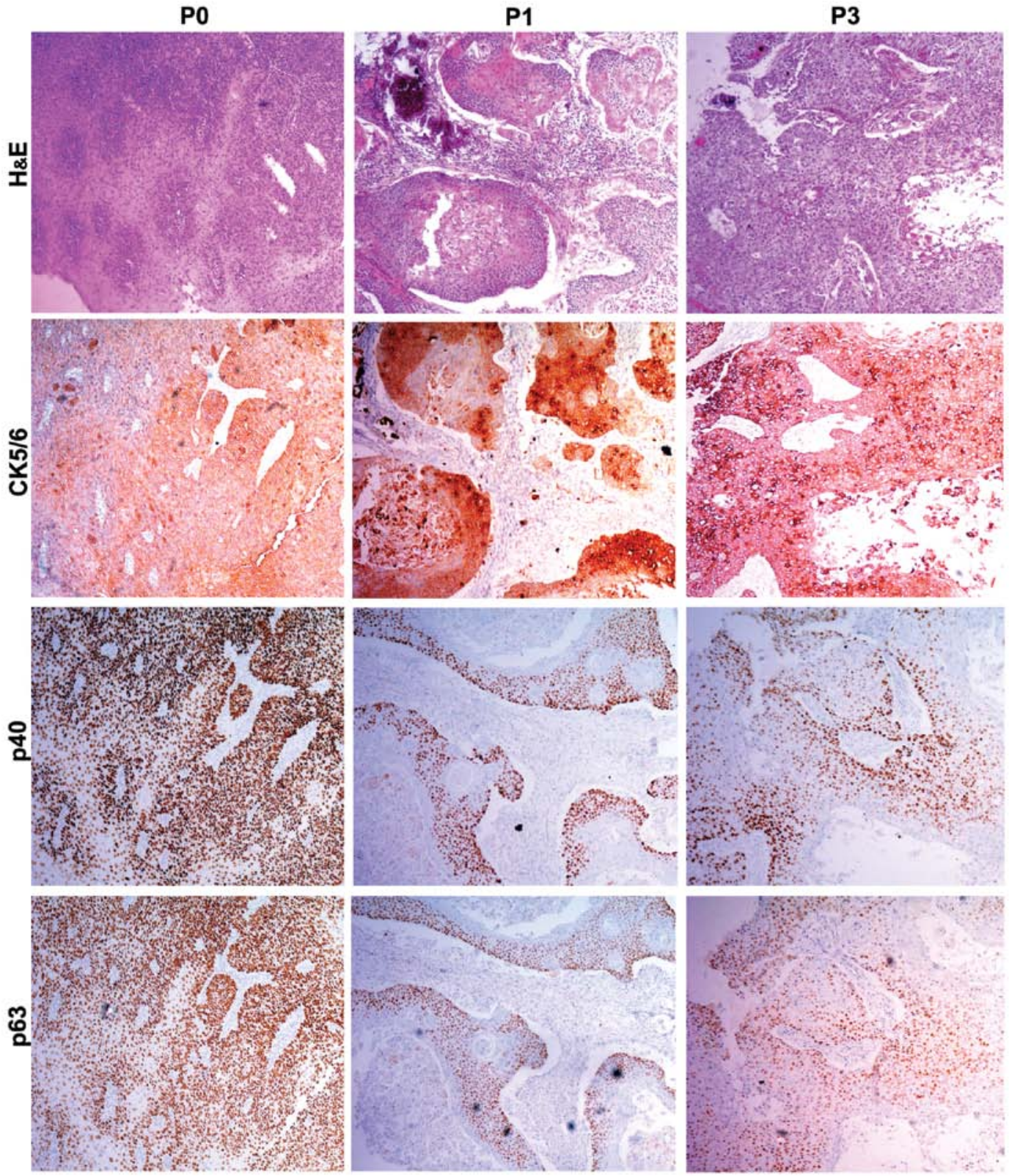

Figure 3. Comparative histology of the original patient tumors with first passage and third passage xenografts for sample EG3. The top row images show histological sections (H\&E) of the esophageal patient tissue (P0), the first (P1) and the third passage (P3) xenograft tissues in the SCID mice. The second, third and last row of images show immunohistochemistry for CK5/6, p40 and p63 in the esophageal patient tissue (P0) and the first (P1) and third passage (P3) xenograft tissues, respectively (magnification, x100).

patients, 17 patients were stage II, 3 patients were stage III, 2 patients were stage II-III, 3 patients were stage I. One patient was stage I-II. According to lymph node metastasis, 10 samples had node metastasis and 6 samples were established, 16 samples had no node metastasis and 8 samples were established. Clinical data of all the patients are shown in Table I.

Growth of ESCC xenografts in SCID mice. Fourteen transplantable xenografts were established, and the success rate was 53.8\%. EG2, EG3 and EG5 were chosen to assess the pathological characteristics. The growth curves of passage three xenografts (first, second and third passage) were plotted as tumor volume over time (Fig. 1). The growth time of the first passage was 123, 108 and 83 days respectively, the second passage was 90, 74 and 81 days, and third passage was 62 , 52 and 72 days. Every group period was shorter in the third passage than the first and second passage. In addition, the growth was stable after the third passage.

Comparison of the histology and immmunohistochemistry between the xenografts and the patient tumors. In order to further evaluate the PDX xenografts, we compared the histology of the original patient tumors with the first and third passage xenografts for samples EG2, EG3 and EG5. We found that the pathological characteristics of the third passage xenografts were in accordance with the original patient samples. The expression of CK5/6, p40 and p63 was positive in the three passages by immunohistochemical staining (Figs. 2-4). 
P0
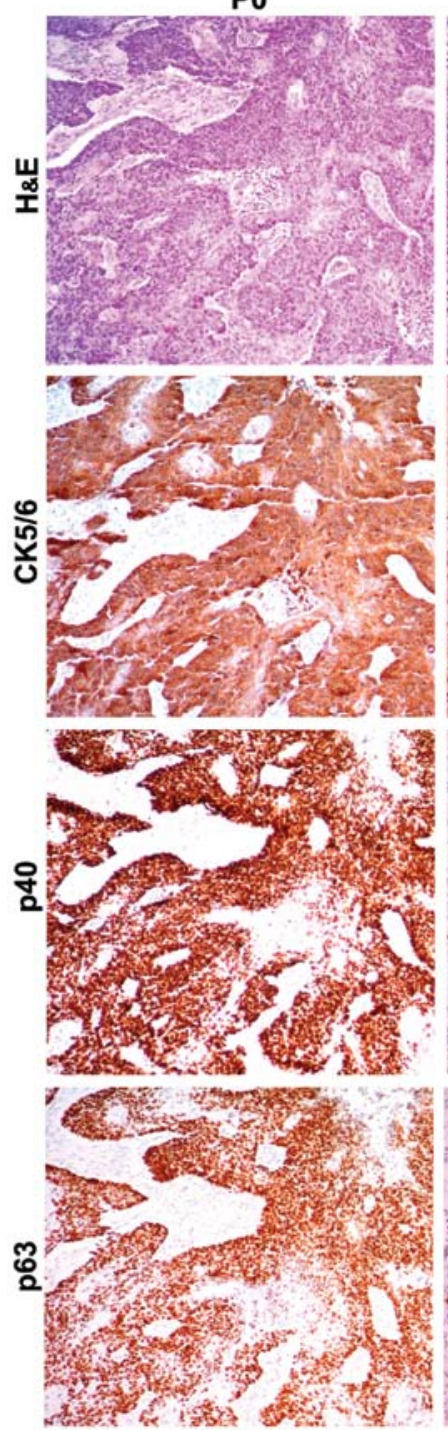

P1

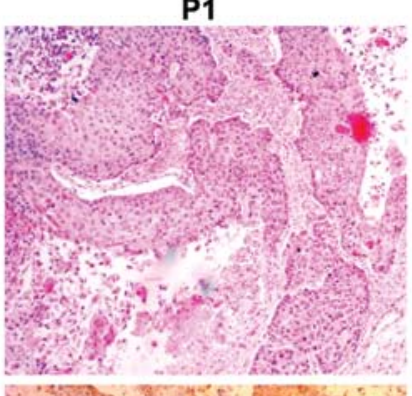

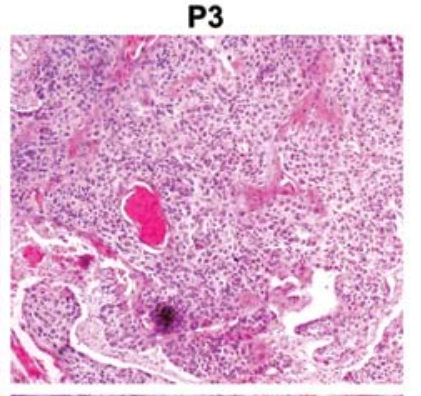
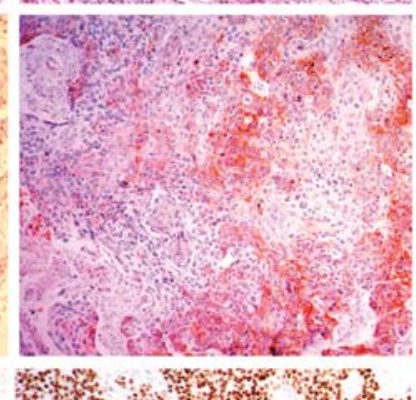
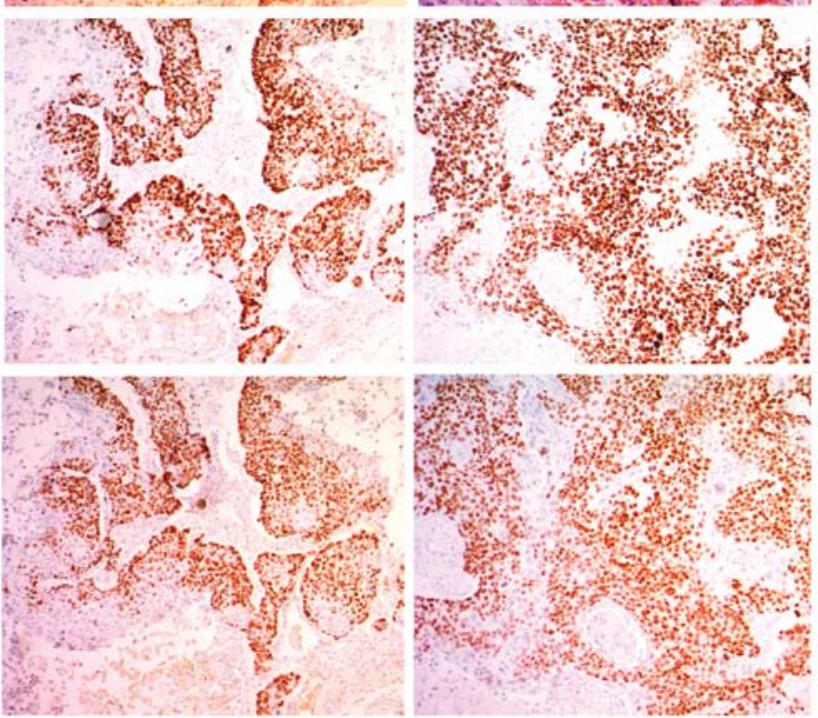

Figure 4. Comparative histology of the original patient tumors with first passage and third passage xenografts for sample EG3. The top row images show histological sections (H\&E) of the esophageal patient tissue (P0), the first (P1) and the third passage (P3) xenograft tissues in the SCID mice. The second, third and last row of images show immunohistochemistry for CK5/6, p40 and p63 in the esophageal patient tissue (P0) and the first (P1) and third passage (P3) xenograft tissues, respectively (magnification, x100).

Activated signal transduction pathways in the established esophageal tumor xenografts. The levels of phosphorylated and total proteins of AKT-mTOR, Stat 3 and Erk 1/2 were detected by western blotting. The level of p-mTOR (Ser2481) was higher in EG8 and EG9 than in EG2, EG3 and EG5, however, p-Akt (Ser473) was higher in EG9 than in EG2, EG3, EG5 and EG8 (Fig. 5A). The level of p-Stat3 (Tyr705) was highest in EG3, however, the level of p-Stat1 (Tyr701) was higher in EG3 and EG8 (Fig. 5B). Moreover, p-ERK1/2 (Thr202/Tyr204) was highest in EG3 (Fig. 5C). The difference in activated signal transduction pathways in these PDXs had statistical significance $(\mathrm{P}=0.000)$.

\section{Discussion}

Esophageal cancer (EC) is a serious malignancy with a high rate of mortality and poor prognosis. While many other types of cancer are expected to decrease in incidence over the next 10 years by 2025, the prevalence of EC is expected to increase by $140 \%$ (12). ESCC is the most common histological type of EC, with a high incidence in China (13). Current clinical treatment strategies for ESCC include surgery, chemotherapy and radiotherapy. Recently, neoadjuvant chemoradiation followed by surgery has been used in the treatment of EC in the clinic (14). Unfortunately, the 5-year survival rate has not improved. Therefore, it is necessary to develop effective therapeutic strategies for ESCC patients.

Animal models are the most useful tool for preclinical evaluation of novel therapeutic strategies in cancer. PDX models can reproduce tumor development, proliferation and metastasis (11) and can predict phase II clinical trial performance (15). Therefore, PDX models play an important role in developing personalized treatment for EC patients (14). In the present study, we established 26 ESCC PDXs subcutaneously. In addition, the success 'take-rate' of the xenografts was 53.8\% and it was higher compared to other EC PDX models for which the success rate was $38.5 \%$ (16). We further evaluated the established PDX models represented as EG2, EG3 and EG5. The 
A
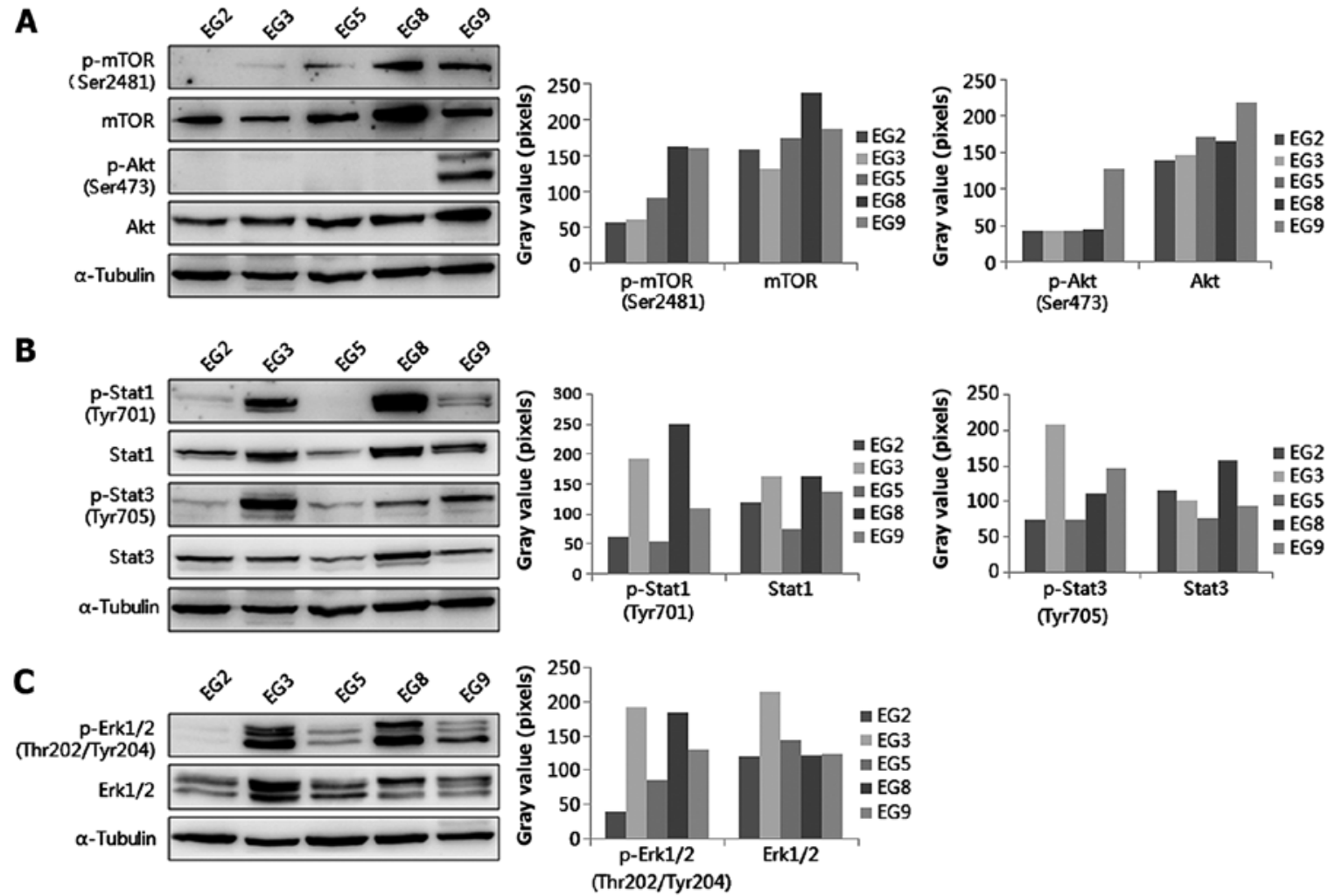

Figure 5. Activated signal transduction pathways [(A) Akt-mTOR axis, (B) Stat1 and Stat3], and (C) Erk1/2 in the established esophageal tumor xenografts. Right, the levels of phosphorylated and total proteins of the Akt-mTOR axis, Stat1 and Stat3, Erk are shown by western blotting. Each experiment was repeated three times. Left histograms, the results of western blotting. ImageJ was used to determine the gray value of the blots, and construct histograms using the gray value. SPSS 17.0 was used to analyze the data; $\mathrm{P}=0.000$, statistical significant difference.

three established ESCC xenografts showed decreased degree of tumor growth curves from first passage to third passage (Fig. 1). In the three PDX models, the first passage reached $1,500 \mathrm{~mm}^{3}$ on day 133, 106 and 75 for EG2, EG3 and EG8, respectively. Next, we performed histology and growth curve analyses of the established xenografts. Expression of CK5/6, p63 and p40 was assessed by immunohistochemsistry. Cytokeratin 5/6 is useful for differentiating epithelioid mesotheliomas from pulmonary carcinomas (17). P63 protein plays important roles in the carcinogenesis of ESCC through the Akt signaling pathway (18). An investigation into the combined expression of p63 may be helpful in early diagnosis and in evaluating the prognosis of ESCC (19). In our study, histological analysis and immunohistochemical analysis were used for assessing the characteristics of the PDX tumors. Expression of CK5/6, p63, p40 in patient samples was positive in the patient tissue and in the first and third passages. Our results indicated that the ESCC PDX models maintained the histological characteristics and the differentiation status of the original patient tumor. In a word, we successfully established ESCC PDX models from patient minimal surgical tissue. The established ESCC PDX models provide a superior in vivo preclinical platform to explore novel cancer therapeutics and analysis of drug activity, including the discovery of predictive biomarkers, the study of ESCC cancer stem-cell biology and stromal-tumor interactions in the clinic.

The drugs used for ESCC patient treatment are toxic to cells and have many side-effects. More effective and innovative treatment strategies are urgently needed (3). However, the molecular tumorigenesis mechanisms of ESCC have not been fully elucidated. Many signaling pathways, for example Akt/mTOR, Erk1/2, Stat1 and Stat3 are involved in the processes of cell growth, cell cycling, apoptosis and invasion in ESCC (20-24). In the present study, we examined the expression of Akt and p-Akt (Ser473), mTOR and p-mTOR in five PDX models. Our results found that the level of p-Akt (Ser473) was positive only in EG9. Our results showed that all groups expressed total mTOR, however, the level of p-mTOR (Ser2481) was higher in EG8 and EG9 than in EG2 and EG5. Thus, we chose the activated Akt/mTOR pathway from our established PDX models to further research the role of the Akt/mTOR pathway in ESCC. The extracellular signal-regulated kinase (Erk1/2) signaling pathway is important for the regulation of cell growth by controlling transcription $(25,26)$. In addition, p-Erk1/2 (Thr202/Tyr204) is higher in poorly differentiated tissues than in well and moderately differentiated tissues in $\operatorname{ESCC}(25,27)$. Our results indicated that Erk1/2 was activated in EG2 compared to the other patients. In addition, its levels were different in these five patient models.

Signal transducer and activator of transcription-3 (Stat3) plays a role in esophageal carcinogenesis by promoting cell proliferation, motility and suppression of apoptosis (28). In addition, high p-Stat3 (Tyr705) is correlated with poor prognosis in ESCC (29). However, Stat1 has opposing biological effects compared with Stat3. The loss of Stat 1 expression significantly correlates with a worse clinical outcome in ESCC (30). Recent research found that Stat1 downregulates Stat 3 and p-Stat 3 (Tyr705), and the relative proportions of Stat1:Stat3 heterodimers decide the cell fate in ESCC (31). 
In our study, we found that $\mathrm{p}$-Stat 1 and $\mathrm{p}$-Stat 3 were overexpressed in EG3, in contrast, there was absence of both in EG2 and EG5. In contrast, p-Stat1 (Tyr701) was lost and p-Stat3 (Tyr705) was activated in EG9. This indicated that the relationship between Stat 3 and Stat1 is complicated. Investigation of the role of Stat1 and Stat 3 and the relationship between both in ESCC are warranted using these xenografts.

In conclusion, we successfully established ESCC patient tumor-derived xenografts. These xenografts maintained the patient's pathological characteristics. They had different growth cycles and tumor texture. Notably, different signaling pathways were activated in ESCC, which reflected the biological heterogeneity observed in the patient population. Furthermore, these PDX models provide an important platform for understanding the molecular mechanisms of ESCC, for elucidating critical signaling pathways involved in esophageal tumorigenesis and progression. These models can also be used to identify potential individual therapeutic targets, investigate the biological activity and toxicity profiles of preclinical drugs and provide a more relevant system to test clinically directed hypotheses in ESCC.

\section{Acknowledgements}

The present study was supported by the National Sciences Foundation of China (nos. 81372269, U1304813 and 81472324), the Science Foundation of the Henan Province of China (nos. 13A310553 and 15B310013), the Young Teacher Special Fund of Zhengzhou University (no. 1421328055) and the Young Teacher Training of Zhengzhou University. We would like to express our thanks to Dr Fred Bogott for critically reading the manuscript and offering good suggestions.

\section{References}

1. Zhao P, Dai M, Chen W and Li N: Cancer trends in China. Jpn J Clin Oncol 40: 281-285, 2010.

2. Adachi $Y$, Ohashi $H$, Imsumran A, Yamamoto H, Matsunaga $Y$, Taniguchi H, Nosho K, Suzuki H, Sasaki Y, Arimura Y, et al: The effect of IGF-I receptor blockade for human esophageal squamous cell carcinoma and adenocarcinoma. Tumour Biol 35 973-985, 2014

3. Belkhiri A and El-Rifai W: Advances in targeted therapies and new promising targets in esophageal cancer. Oncotarget 6 : $1348-1358,2015$

4. Honjo S, Ajani JA, Scott AW, Chen Q, Skinner HD, Stroehlein J, Johnson RL and Song S: Metformin sensitizes chemotherapy by targeting cancer stem cells and the mTOR pathway in esophageal cancer. Int J Oncol 45: 567-574, 2014.

5. Harb WA: Management of patients with hormone receptorpositive breast cancer with visceral disease: Challenges and treatment options. Cancer Manag Res 7: 37-46, 2015.

6. Geuna E, Milani A, Martinello R, Aversa C, Valabrega G Scaltriti M and Montemurro F: Buparlisib, an oral pan-PI3K inhibitor for the treatment of breast cancer. Expert Opin Investig Drugs 24: 421-431, 2015.

7. Inoue A, Saijo Y, Maemondo M, Gomi K, Tokue Y, Kimura Y, Ebina M, Kikuchi T, Moriya T and Nukiwa T: Severe acute interstitial pneumonia and gefitinib. Lancet 361: 137-139, 2003.

8. Baselga J, Rischin D, Ranson M, Calvert H, Raymond E, Kieback DG, Kaye SB, Gianni L, Harris A, Bjork T, et al: Phase I safety, pharmacokinetic, and pharmacodynamic trial of ZD1839, a selective oral epidermal growth factor receptor tyrosine kinase inhibitor, in patients with five selected solid tumor types. J Clin Oncol 20: 4292-4302, 2002.

9. Marangoni E, Vincent-Salomon A, Auger N, Degeorges A, Assayag F, de Cremoux P, de Plater L, Guyader C, De Pinieux G, Judde JG et al: A new model of patient tumor-derived breast cancer xenografts for preclinical assays. Clin Cancer Res 13: 3989-3998, 2007.
10. Moro M, Bertolini G, Tortoreto M, Pastorino U, Sozzi G and Roz L: Patient-derived xenografts of non small cell lung cancer: Resurgence of an old model for investigation of modern concepts of tailored therapy and cancer stem cells. J Biomed Biotechnol 2012: 568567, 2012.

11. Siolas D and Hannon GJ: Patient-derived tumor xenografts: Transforming clinical samples into mouse models. Cancer Res 73: 5315-5319, 2013.

12. Tian H, Hou L, Xiong YM, Huang JX, She YJ, Bi XB and Song XR: miR-218 suppresses tumor growth and enhances the chemosensitivity of esophageal squamous cell carcinoma to cisplatin. Oncol Rep 33: 981-989, 2015.

13. Napier KJ, Scheerer M and Misra S: Esophageal cancer: A Review of epidemiology, pathogenesis, staging workup and treatment modalities. World J Gastrointest Oncol 6: 112-120, 2014.

14. Uemura $\mathrm{N}$ and Kondo T: Current status of predictive biomarkers for neoadjuvant therapy in esophageal cancer. World J Gastrointest Pathophysiol 5: 322-334, 2014.

15. Morton CL and Houghton PJ: Establishment of human tumor xenografts in immunodeficient mice. Nat Protoc 2: 247-250, 2007.

16. Dong SW, Zhang H, Wang BL, Sun P, Wang YG and Zhang P: Effect of the downregulation of SMYD3 expression by RNAi on RIZ1 expression and proliferation of esophageal squamous cell carcinoma. Oncol Rep 32: 1064-1070, 2014.

17. Blobel GA, Moll R, Franke WW, Kayser KW and Gould VE: The intermediate filament cytoskeleton of malignant mesotheliomas and its diagnostic significance. Am J Pathol 121: 235-247, 1985.

18. Ye S, Lee KB, Park MH, Lee JS and Kim SM: p63 regulates growth of esophageal squamous carcinoma cells via the Akt signaling pathway. Int J Oncol 44: 2153-2159, 2014.

19. Thépot A, Hautefeuille A, Cros MP, Abedi-Ardekani B, Pétré A, Damour O, Krutovskikh $\mathrm{V}$ and Hainaut P: Intraepithelial p63-dependent expression of distinct components of cell adhesion complexes in normal esophageal mucosa and squamous cell carcinoma. Int J Cancer 127: 2051-2062, 2010.

20. Lin DC, Hao JJ, Nagata Y, Xu L, Shang L, Meng X, Sato Y, Okuno Y, Varela AM, Ding LW, et al: Genomic and molecular characterization of esophageal squamous cell carcinoma. Nat Genet 46: 467-473, 2014.

21. Song Y, Li L, Ou Y, Gao Z, Li E, Li X, Zhang W, Wang J, Xu L, Zhou Y, et al: Identification of genomic alterations in oesophageal squamous cell cancer. Nature 509: 91-95, 2014.

22. Li B, Li J, Xu WW, Guan XY, Qin YR, Zhang LY, Law S, Tsao SW and Cheung AL: Suppression of esophageal tumor growth and chemoresistance by directly targeting the PI3K/AKT pathway. Oncotarget 5: 11576-11587, 2014.

23. Hou G, Yang S, Zhou Y, Wang C, Zhao W and Lu Z: Targeted inhibition of mTOR signaling improves sensitivity of esophageal squamous cell carcinoma cells to cisplatin. J Immunol Res 2014: 845763, 2014.

24. Huang Y, Xi Q, Chen Y, Wang J, Peng P, Xia S and Yu S: A dual mTORC1 and mTORC2 inhibitor shows antitumor activity in esophageal squamous cell carcinoma cells and sensitizes them to cisplatin. Anticancer Drugs 24: 889-898, 2013.

25. Ciccarelli A and Giustetto M: Role of ERK signaling in activity-dependent modifications of histone proteins. Neuropharmacology 80: 34-44, 2014.

26. Bhalla S, Evens AM, Dai B, Prachand S, Gordon LI and Gartenhaus RB: The novel anti-MEK small molecule AZD6244 induces BIM-dependent and AKT-independent apoptosis in diffuse large B-cell lymphoma. Blood 118: 1052-1061, 2011.

27. Zhang J, Zhi H, Zhou C, Ding F, Luo A, Zhang X, Sun Y, Wang X, Wu M and Liu Z: Up-regulation of fibronectin in oesophageal squamous cell carcinoma is associated with activation of the Erk pathway. J Pathol 207: 402-409, 2005.

28. Sakamoto C: STAT1 and STAT3 might be regulated differently in esophageal squamous cell carcinoma. J Gastroenterol 37: 575-577, 2002.

29. Wang Z, Zhu S, Shen M, Liu J, Wang M, Li C, Wang Y, Deng A and Mei Q: STAT3 is involved in esophageal carcinogenesis through regulation of Oct-1. Carcinogenesis 34: 678-688, 2013.

30. Qing Y and Stark GR: Alternative activation of STAT1 and STAT3 in response to interferon-gamma. J Biol Chem 279: 41679-41685, 2004.

31. Regis G, Pensa S, Boselli D, Novelli F and Poli V: Ups and downs: the STAT1:STAT3 seesaw of Interferon and gp130 receptor signalling. Semin Cell Dev Biol 19: 351-359, 2008. 